\title{
Advances in dynamic temporal networks: Understanding the temporal dynamics of complex adaptive networks
}

\author{
Peter M.A. Sloot ${ }^{1,2,3, a}$, George Kampis ${ }^{4,6,7, b}$, and László Gulyás ${ }^{4,5,6, c}$ \\ 1 University of Amsterdam, The Netherlands \\ 2 Research Institute ITMO, St. Petersburg, Russian Federation \\ 3 Complexity Program, Nanyang Technological University, Singapore \\ 4 Lorand Eötvös University, Budapest, Hungary \\ 5 Aitia International, Inc., Budapest, Hungary \\ 6 PetaByte Research Ltd., Budapest, Hungary \\ 7 DFKI (German Research Insitute for Artificial Intelligence), Trippstadter Strasse 122, \\ 67663 Kaiserslautern, Germany
}

Received 3 June 2013 / Received in final form 10 July 2013

Published online 13 September 2013

\begin{abstract}
Complex networks have been extensively studied in the past 15 years and with increasing details. However, research on the temporal dynamics of complex networks is largely a new territory yet to map out. The present volume presents a collection of papers dealing with various aspects of the problem and this editorial introduces the field as well as the papers.
\end{abstract}

\section{Introduction}

Over the past few years we have come to realize that networks are all around us. Even we are ourselves, as individuals, the units of a network of social relationships of different kinds and, as biological systems, the delicate result of a network of biochemical reactions. Networks can be tangible objects in the Euclidean space, such as electric power grids, the Internet, highways or subway systems, and neural networks. Or they can be entities defined in an abstract space, such as networks of collaborations between individuals.

\section{The theory of complex networks}

The development of the theory of complex networks (including social networks and systems from other domains) has been in the foreground of interest in the past decade.

\footnotetext{
a e-mail: p.m.a.sloot@uva.nl

b e-mail: gk@hps.elte.hu

c e-mail: gulyas@petabyte-research.org
} 
Many network characteristics were identified and several network models introduced. However, the overwhelming majority of these have dealt with networks understood as static objects, unchanging in time.

For example, the various well-known measures describing important structural properties of the network, such as diameter (largest distance between two nodes of the network), path length (average distance between the nodes), or the various forms of centrality (i.e., node importance) are all defined on invariant, and by that virtue, static networks. Similarly, the study of the most important statistical properties such as degree distributions (characterizing the number of neighbors nodes have) assume the same form. Real world networks are, however, inherently dynamic, as virtually no natural network pops up instantaneously and in full form. A few issues of network generation have been dealt with by network growth models (e.g. $[2,3,32]$ ), but the aim of these now-classical works was to characterize properties of the resulting network (i.e. the envelope), and not the dynamic temporal process itself. Also, in real networks the changes often occur for endogenous, network-internal reasons that are more complicated than can be grasped by a simple growth rule. For instance, the change of a node's internal attributes can imply changes in its connectivity, and so on. (In an epidemic network, the recovered patient does not infect any more, so no more infection links can be formed from this node.) Interests of this kind put the questions of the interrelated dynamics of and on networks (e.g. [19,24,26,33]) into a new focus of investigation. Several important problems ranging from virology [4], to contact networks [34,35] and ecological theory [23] similarly invite a treatment of dynamic temporal networks on their own. A list of early works is given in $[10]$.

\section{Towards dynamic networks}

Partly as a response to these current challenges, several approaches and tools were introduced more recently $[18,25,40]$. These are mostly isolated works, however, focusing on special problems. Only a few aim at developing general methodology. The SONIA project of Stanford [5] promises the visualization of longitudinal network data, but also raises some of the most important methodological questions for general dynamic networks. New problems include that of slicing, i.e. the use of thin or thick time slices or windows, and the implied question of how a flexible binning (the grouping of raw data into varying time slices) is achievable to obtain relevant network properties at a zero width snapshot the network properties, at a full time window, the dynamics is lost [31]. SONIA is built on the assumption that time stamped and -sliced data will grow in significance. Yet public longitudinal network data are still rare (a comprehensive list is [37]).

Dynamic Network Analysis (DNA, [9]) is the name of a prolific approach to various Social Network Analysis problems (the general name is slightly misleading, as DNA is more about modeling and decision support; also, DNA considers special nodes which, just like human agents, can learn). DNA uses the meta-matrix concept [9] to grasp multi-mode, multiplex, dynamic networks with multiple node attributes (such as a person's relation to events, organizations, resources, and other people). Some radically new network measures such as the notion of "cognitive load" were introduced - this is the amount of information that an agent has to handle in order to maintain its position in the network, and translates to connection intensity in a multi-net. DNA also endeavored to develop an interoperable dynamic network toolkit [11]. All in all, this is an interesting endeavor based on the recognition of the entirely new kinds of problems encountered in the dynamic domain, but it does not alone change the status of the field. 
Recently, an important new area of research deals with extending the basic structural measures to dynamically changing temporal networks [21]. Our aim is to continue this new line with original contributions.

\section{Complex temporal dynamics of networks}

To motivate our approach, note that many relevant questions arise when studying complex networks' dynamics, such as understanding how a large ensemble of dynamical systems that interact through a complex wiring topology can behave collectively. Networks evolve over time and show a remarkable robustness and resilience to "environmental pressure". Examples are Protein-Protein Networks [15], Slime Mold Networks [1], Phylogenetic-Social Networks [41], Sexual Networks [28,29,35], "Bio" networks [22], Drug Addiction Networks [16] and Criminal Networks [17]. This robustness and resilience is often realized through dynamic modification of the network topology as well as the behavioural and functional changes of the links and nodes. Here we avoid the word "optimization" since these systems seem to function way out of equilibrium and the notion of "optimal" might not apply.

The dynamics on and of the network may emerge from a variety of causes. Yet, they seem to lead to specific classes of behavior. An important aspect seems to be the resilience with respect to uncertainty and noise. For instance it has recently been shown that stochastic resonance in the presence of noise can actually enhance information transfer in hierarchical complex social networks [14]. It seems likely that this resilience in complex networks has emerged from "evolutionary" adaptation, but little is known about that: it is an open research area.

In order to quantify, understand and even predict complex phenomena in a networked world, we need to combine the expressiveness of Individual Based Models with the structural information of Networks and quantify the way information flows through it a network $[20,30,36,38]$.

\section{Papers in this issue}

This special issue on "Advances in Dynamic Temporal Networks" monitors the developments in this rapidly changing field, using 11 original invited contributions by leading experts.

The first two contributions discuss the dynamics of networks:

- Barrat et al. [42] studies empirical temporal networks of face to face human interactions using their original technique to apply RFID counters to register temporary human-to-human interactions in open spaces;

- Gulyás et al. [43] develop new theoretical models to understand the universal baseline dynamics of the temporal evolution of networks. This is the first systematic study of the elementary dynamics of edge addition and deletion using different classes of operators and varying aggregation windows.

The second section deals with the more classic issue of dynamics on the network and thus contains a single paper. The remarkable contribution of

- Czaplicka et al. [44] applies the theory of stochastic resonance to studying information flow in hierarchical networks.

The third section combines the approaches of the first two. It contains contributions tackling the combination of the two above types of network dynamics: dynamics on and of networks:

- Quax et al. [45] infers epidemiological parameters from phylogenetic information using this novel approach. On the other hand, 
- Gulyás and Kampis [46] develops an agent-based model of spreading processes on dynamically changing contact networks. Finally,

- Zarrabi et al. [47] combines social and genetic network dynamics to study HIV transmission.

This is followed by an important paper developing the information theory of dynamics transmissions on networks, which served as the basis of a recent Ph.D. thesis:

- by Quax et al. [48] presenting a novel information theory of dynamics networks.

The volume is concluded with a chapter on applications. Four contributions use the dynamic temporal network approach in different problem domains.

- van de Vijver et al. [49] investigate the transmission of HIV in sexual networks in sub-Saharan Africa and Europe.

- Tóth et al. [50] develop novel centrality measures to grasp the dynamics of value chains in dark networks and test these new theoretical tools on classic (non-dark) empirical networks.

- The last two contributions take the volume's approach to the domain of scientometrics. Soós et al. [51] process extremely large volumes of information when performing the temporal analysis of the entire history of computer and information science using the temporal network approach.

- Apolloni et al. [52], finally, investigate the spatio-temporal relationships of scientific collaboration.

Together, elements of this collection show a variety of aspects of the current study field of dynamic, temporal networks. An essential recognition, that dynamic temporal networks behave differently from their static counterparts, emerges and is reiterated in several of the results. This is true for general information spreading but also for the study of infections, yielding the counter-intuitive results that preferential attachment networks are not the fastest spreaders of an infection - but also reflected in the human-to-human contact studies showing the importance of time windows over degree distributions. There is a host of future works expected along these lines and the current collection can only give a present cross-section of them. We hope that with the above contributions, the issue will inspire researchers in all fields of science to study and exploit the expressiveness of dynamic complex networks.

The work is supported by DynaNets. DynaNets acknowledges the financial support of the Future and Emerging Technologies (FET) program within the Seventh Framework Program for Research of the European Commission, under FET-Open grant number: 233847. PMAS also acknowledges the support from a grant from the "Leading Scientist Program" of the Government of the Russian Federation, under contract 11.G34.31.0019 and the support from the FET-Proactive grant TOPDRIM, number FP7-ICT-318121 as well as the Complexity program of the NTU in Singapore.

\section{References}

1. A. Adamatzky, M.H. Lees, P.M.A. Sloot, Adv. Complex Syst. 15, 1250034+28, doi: $10.1142 / \mathrm{S} 0219525912500348$ (2012)

2. A.-L. Barabasi, Science 325, 412 (2009)

3. A.-L. Barabasi, R. Albert, Science 286, 5439 (1999)

4. A. Barrat, M. Barthelemy, A. Vespignani, Dynamical processes in complex networks (Cambridge University Press, 2008) 
5. S. Bender-deMoll, D.A. McFarland, J. Soc. Struct. 7, 2 (2006)

6. K. Börner, Plug-and-Play Macroscopes (Comm. ACM, 2009) (in press)

7. K. Börner, Ch. Chen, K.W. Boyack, Visualizing Knowledge Domains, in: Annual Review of Information Science 8 Technology (ARIST) edited by Blaise Cronin, Vol. 37, Medford, NJ: Information Today, Inc./American Society for Information Science and Technology (2003), p. 179

8. K. Börner, B.W.II. Herr, J.-D. Fekete, IV07 Software Infrastructures Workshop. Paper presented at the 11th International Conference on Information Visualization (Zurich, Switzerland, 2007)

9. K. Carley, Dynamic Network Analysis. Committee on Human Factors, National Research Council (2003), p. 133

10. K.M. Carley, Dynamic network analysis. In Dynamic social network modeling and analysis: Workshop summary and papers. Comittee on Human Factors, National Research Council (2003), p. 133

11. K. Carley, J. Diesner, J. Reminga, M. Tsvetovat, Toward an Interoperable Dynamic Network Analysis Toolkit, DSS Special Issue on Cyberinfrastructure for Homeland Security (2005)

12. C. Chen, J. Amer. Soc. Inf. Sci. Technol. 57, 359 (2006)

13. C. Chen, Proc. Nat. Acad. Sci. USA (PNAS), 101 (Suppl. 1), 5303 (2004)

14. A. Czaplicka, J.A. Holyst, P.M.A. Sloot, Noise enhances information transfer in hierarchical networks. Nature Science Reports Scientific Reports 3, Article number: 1223 doi: 10.1038/srep01223, Feb. (2013)

15. D. van Dijk, G. Ertaylan, C.A.B. Boucher, P.M.A. Sloot, Identifying potential survival strategies of HIV-1 through virus-host protein interaction networks, BMC Systems Biology, (PubMedID 20633292) Vol. 4, ISSN 1752-0509, DOI: 10.1186/1752-0509-4-96 http://www.biomedcentral.com/1752-0509/4/96 (2010), p. 96

16. L.J. Dijkstra, A.V. Yakushev, P.A.C. Duijn, A.V. Boukhanovsky, P.M.A. Sloot, Inference of the Russian drug community from one of the largest social networks in the Russian Federation. Quality \& Quantity, http://arxiv.org/abs/1211.4783 (in press) (2013)

17. P. Duijn, V. Kashirin, P.M.A. Sloot, The Relative Ineffectiveness of Dark Network Disruption Strategies (submitted)

18. T. Falkowski, J. Bartelheimer, M. Spiliopoulou, Mining and visualizing the evolution of subgroups in social networks, in: 2006 IEEE/WIC/ACM International Conference on Web Intelligence (WI 2006 main conference proceedings) (2006)

19. N. Ganguly, A. Deutsch, A. Mukherjee (eds.), Dynamics On and Of Complex Networks. Applications to Biology, Computer Science, and the Social Sciences. Series: Modeling and Simulation in Science, Engineering and Technology (Birkhauser, New York, 2009)

20. A.G. Hoekstra, J. Kroc, P.M.A. Sloot (eds.), Simulating Complex Systems by Cellular Automata, in series Understanding Complex Systems, 384 pages (Springer, 2010), ISBN: 978-3-642-12202-6

21. P. Holme, J. Saramäki, Temporal Networks, Physics Reports 509, 97 (2012), ISSN 0370-1573, doi: 10.1016/j.physrep.2012.03.001, http://www.sciencedirect.com/ science/article/pii/S0370157312000841

22. S. Jaeger, G. Ertaylan, D. van Dijk, U. Leser, P.M.A. Sloot: Inference of Surface Membrane Factors of HIV-1 Infection through Functional Interaction Networks, PLoS One, Vol. 5, pp. e13139+12, doi: 10.1371/journal.pone.0013139, http://dx.doi.org/10.1371\%2Fjournal.pone.0013139 (2010)

23. F. Jordán, I. Scheuring, Phys. Life Rev. 1, 139 (2004)

24. G. Kossinets, D.J. Watts, Empirical Analysis of an Evolving Social Network, Science 311, 88 (2006) doi: 10.1126/science.1116869, http://www.sciencemag.org/ content/311/5757/88.full.pdf

25. M. Lahiri, T.Y. Berger-Wolf, Mining Periodic Behavior in Dynamic Social Networks, Eighth IEEE International Conference on Data Mining, ICDM '08 (2008), p. 373

26. J. Leskovec, Dynamics of large networks, Ph.D. Dissertation, Machine Learning Department, School of Computer Science, Carnegie Mellon University, Technical report CMU-ML-08-111, September (2008) 
27. L. Leydesdorff, J. Amer. Soc. Inf. Sci. Technol. 58, 1303 (2007)

28. S. Mei, P.M.A. Sloot, R. Quax, Y. Zhu, W. Wang, Complex Agent Networks explaining the HIV epidemic among homosexual men in Amsterdam, Mathematics and Computers in Simulation, Vol. 80, p. 1018 (2010), doi: 10.1016/j.matcom.2009.12.008, http://dx.doi.org/10.1016/j.matcom.2009.12.008

29. S. Mei, R. Quax, D.A.M.C. van de Vijver, Y. Zhu, P.M.A. Sloot, Increasing risk behaviour can outweigh the benefits of antiretroviral drug treatment on the HIV incidence among men-having-sex-with-men in Amsterdam, BMC Infectious Diseases, Vol. 11, 17 pages, doi: 10.1186/1471-2334-11-118, http://www.biomedcentral.com/1471-2334/11/118 (2011)

30. S. Mei, N. Zarrabi, M. Lees, P.M.A. Sloot, Complex Agent Networks: An Emerging approach for Modeling Complex Systems, Simulation Modelling Practice and Theory, (in press) (2013)

31. J. Moody, D.A. McFarland, S. Bender-deMoll, Amer. J. Sociol. (2005)

32. D.M. Pennock, G.W. Flake, S. Lawrence, E.J. Glover, C.L. Giles, Winners Don't Take All: Characterizing the Competition for Links on the Web. PNAS 99, 5207 (2002)

33. C. Shalizi, M.F. Camperi, K.L. Klinkner, edited by A. Goldenberg, E. Airoldi, S.E. Fienberg, A. Zheng, D.M. Blei, E.P. Xing, Statistical Network Analysis: Models, Issues and New Directions (New York: Springer-Verlag, 2007), p. 140157

34. P.M.A. Sloot, V. Peter Coveney, G. Ertaylan, V. Muller, C.A. Boucher, M. Bubak, Phil. Trans. R. Soc. A 367, 2691 (2009)

35. P.M.A. Sloot, S.V. Ivanov, A.V. Boukhanovsky, D.A.M.C. van de Vijver, C.A.B. Boucher, Int. J. Comp. Math. Vol. 85 (Taylor \& Francis, 2008), doi: 10.1080/00207160701750583 http://www.science.uva. nl/research/pscs/papers/archive/Sloot2008d.pdf

36. P.M.A. Sloot, R. Quax, Information processing as a paradigm to model and simulate complex systems, J. Comput. Sci., pp. 247 (2012), ISSN: 1877-7503, doi: 10.1016/j.jocs.2012.07.001, http://www.sciencedirect.com/ science/article/pii/S1877750312000725

37. Longitudinal Network Data Sets at Stanford, http://www.stanford.edu/group/sonia/ dataSources/index.html

38. R. Quax, A. Apolloni, P.M.A. Sloot, The diminishing role of highly connected units in the dynamical behaviour of complex networks, Journal of the Royal Society Interface, (in press), (2013) http://arxiv.org/abs/1111.5483

39. Z. Szakolczi, A tudománymetriai vizsgálatok során előálló, időben változó hálózatsorozatok elemzése, MSc. thesis (in Hungarian), 2009

40. M. Trier, A. Bobrik, Analyzing the Dynamics of Community Formation using Brokering Activities, In: Proceedings of the Third Communities and Technologies Conference (Michigan, Springer Series, 2007)

41. N. Zarrabi, M.C.F. Prosperi, R.G. Belleman, M. Colafigli, A. De Luca, P.M.A. Sloot, Combining Epidemiological and Genetic Networks Signifies the Importance of Early Treatment in HIV-1 Transmission, PLoS One, Vol. 7, e46156+15. Public Library of Science, 09 (2012), http://www.plosone.org/article/ info\%3Adoi $\% 2$ F $10.1371 \% 2 \mathrm{Fjournal}$. pone. 0046156

42. A. Barrat, C. Cattuto, V. Colizza, F. Gesualdo, L. Isella, E. Pandolfi, J.-F. Pinton, L. Rava, C. Rizzo, M. Romano, J. Stehle, A.E. Tozzi, W. Van den Broeck, Eur. Phys. J. Special Topics 222 (6), 1295 (2013)

43. L. Gulyas, G. Kampis, R.O. Legendi, Eur. Phys. J. Special Topics 222 (6), 1311 (2013)

44. A. Czaplicka, J.A. Holyst, P.M.A. Sloot, Eur. Phys. J. Special Topics 222 (6), 1335 (2013)

45. R. Quax, D.A.M.C. van de Vijver, D. Frentz, P.M.A. Sloot, Eur. Phys. J. Special Topics 222 (6), 1347 (2013)

46. L. Gulyas, G. Kampis, Eur. Phys. J. Special Topics 222 (6), 1359 (2013)

47. N. Zarrabi, M.C.F. Prosperi, R.G. Belleman1, S. Di Giambenedetto, M. Fabbiani, A. De Luca, P.M.A. Sloot, Eur. Phys. J. Special Topics 222 (6), 1377 (2013) 
48. R. Quax, A. Apolloni, P.M.A. Sloot, Eur. Phys. J. Special Topics 222 (6), 1389 (2013)

49. D.A.M.C. van de Vijver, M.C.F. Prosperi, J.J. Ramasco, Eur. Phys. J. Special Topics 222 (6), 1403 (2013)

50. N. Tóth, L. Gulyas, R.O. Legendi, P. Duijn, P.M.A. Sloot, G. Kampis, Eur. Phys. J. Special Topics 222 (6), 1413 (2013)

51. S. Soos, G. Kampis, L. Gulyas, Eur. Phys. J. Special Topics 222 (6), 1441 (2013)

52. A. Apolloni, J.-B. Rouquier, P. Jensen, Eur. Phys. J. Special Topics 222 (6), 1467 (2013) 